\title{
Emerging Roles of RNA-Binding Proteins in Plant Growth, Development, and Stress Responses
}

\author{
Kwanuk Lee, and Hunseung Kang *
}

\begin{abstract}
Posttranscriptional regulation of RNA metabolism, including RNA processing, intron splicing, editing, RNA export, and decay, is increasingly regarded as an essential step for fine-tuning the regulation of gene expression in eukaryotes. RNA-binding proteins (RBPs) are central regulatory factors controlling posttranscriptional RNA metabolism during plant growth, development, and stress responses. Although functional roles of diverse RBPs in living organisms have been determined during the last decades, our understanding of the functional roles of RBPs in plants is lagging far behind our understanding of those in other organisms, including animals, bacteria, and viruses. However, recent functional analysis of multiple $R B P$ family members involved in plant RNA metabolism and elucidation of the mechanistic roles of RBPs shed light on the cellular roles of diverse RBPs in growth, development, and stress responses of plants. In this review, we will discuss recent studies demonstrating the emerging roles of multiple RBP family members that play essential roles in RNA metabolism during plant growth, development, and stress responses.
\end{abstract}

\section{INTRODUCTION}

Gene expression in living organisms is commonly regulated at both transcriptional and posttranscriptional levels, which is crucial for the growth and development as well as the response and adaptation of the organisms to diverse environmental stimuli (Floris et al., 2009; Simpson and Filipowicz, 1996). Posttranscriptional regulation of gene expression includes RNA processing, pre-mRNA splicing, and RNA export and decay, which is referred to as RNA metabolism and is central for various cellular processes in eukaryotes (Simpson and Filipowicz, 1996). The regulation of RNA metabolism is carried out by either direct or indirect binding of RNA-binding proteins (RBPs) to target RNAs. Determination of protein structures and functional characterization of RBPs in diverse organisms have revealed that RBPs harbor several conserved motifs and domains, in-

Department of Plant Biotechnology, College of Agriculture and Life Sciences, Chonnam National University, Gwangju 500-757, Korea

${ }^{*}$ Correspondence: hskang@jnu.ac.kr

Received 23 December, 2015; revised 30 December, 2015; accepted 4 January, 2016; published online 2 February, 2016

Keywords: plant development, RNA-binding protein, RNA chaperone, RNA metabolism, stress response. cluding RNA-recognition motif (RRM), zinc finger motif, $\mathrm{K}$ homology $(\mathrm{KH})$ domain, glycine-rich region, arginine-rich region, RD-repeats, and SR-repeats (Alba and Pages, 1998; Lorković and Barta, 2002). Plant genomes encode a variety of RBPs, which suggests the functional diversity of RBPs in plant growth, development, and stress responses (Lorković, 2009; Mangeon et al., 2010). In particular, those RBPs harboring an RRM at the $\mathrm{N}$-terminus and a glycine-rich region at the C-terminus, thus referred to as glycine-rich RBP (GRP), zinc finger-containing GRP (RZ), cold shock domain protein (CSDP), and RNA helicase $(\mathrm{RH})$ have been implicated to play crucial roles in plant growth and stress responses (Jankowsky, 2011; Jung et al., 2013; Mihailovich et al., 2010). Importantly, many nuclearencoded chloroplast- or mitochondria-targeted RBPs play pivotal roles in RNA metabolism in chloroplasts and mitochondria (Hammani and Giege, 2014; de Longevialle et al., 2010; Stern et al., 2010). Several recent studies have demonstrated that chloroplast-targeted proteins, including $\mathrm{RH}$, chloroplast RNA splicing and ribosome maturation protein (CRM), S1 RNAbinding domain protein (SDP), and pentatricopeptide repeat protein (PPR) participate in rRNA processing or intron splicing under normal and stress conditions (Gu et al., 2014; 2015; Han et al., 2015; Lee et al., 2014). Moreover, plant-specific PPRs are mainly targeted to chloroplasts or mitochondria and are involved in organellar RNA metabolism (Barkan and Small, 2014; Brown et al., 2014; de Longevialle et al., 2010; SchmitzLinneweber and Small, 2008). In this review, we will focus on several RBP families, including GRP, RZ, CSDP, RH, CRM, SDP, and PPR, which play crucial roles in plant growth, development, and stress responses.

\section{STRUCTURAL FEATURES OF RBPS}

RRM is the best-characterized RNA-binding motif found in all eukaryotes, and many RBPs possess one or two RRMs at the N-terminal region (Alba and Pages, 1998; Lorković and Barta, 2002). Typical RBPs contain auxiliary domains or motifs at the $\mathrm{C}$-terminal region, including glycine-rich region, zinc finger motif, arginine-rich domain, RD-repeats, and SRrepeats, which determine the binding specificity of RBPs to target RNAs (Nagai et al., 1995). Among those RBPs, GRPs are well-characterized in plants, and the genomes of Arabidopsis thaliana and rice (Oryza sativa) harbor eight and six GRP genes, respectively (Lorković and Barta, 2002). GRPs contain a canonical RRM at the $\mathrm{N}$-terminus and a glycine-rich region at the C-terminus (Fig. 1). GRPs harboring an additional CCHC-type zinc finger in the glycine-rich region, referred to 


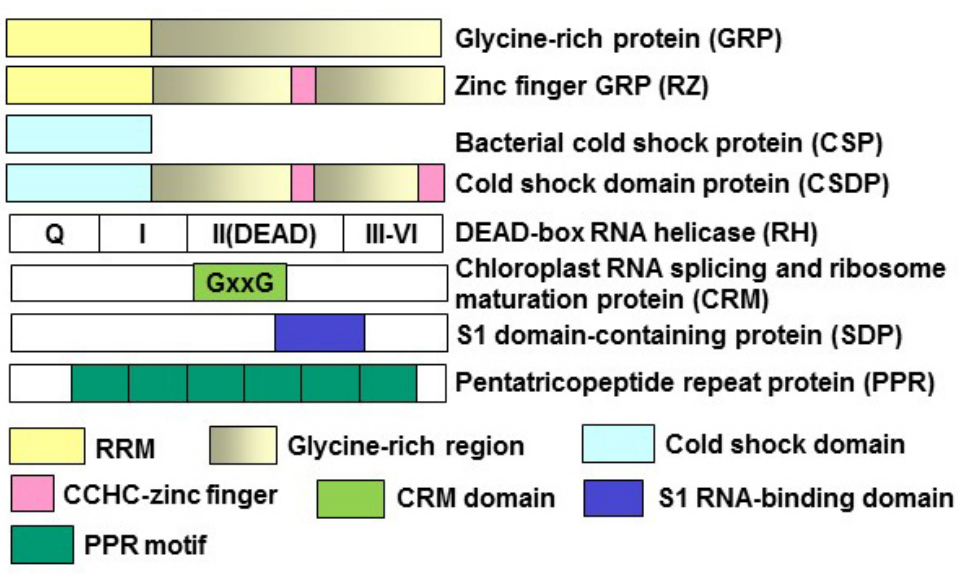

Fig. 1. Schematic presentation of domain structures of RNA-binding proteins. Glycine-rich RNA-binding protein (GRP) harbors an RNA-recognition motif (RRM) at the $\mathrm{N}$-terminal half and a glycine-rich region at the C-terminal half. RZ protein contains an RRM and a glycine-rich region interspersed with a CCHC-type zinc finger motif. Cold shock domain protein (CSDP) harbors an N-terminal cold shock domain (CSD) and a Cterminal glycine-rich region interspersed with a CCHC-type zinc finger motif. DEAD-box RNA helicase (RH) consists of $\mathrm{Q}, \mathrm{I}, \mathrm{II}$ (DEAD), III, IV, V and VI domains. Chloroplast RNA splicing and ribosome maturation domain (CRM) protein contains a highly conserved GXXG sequence, and $S 1$ domain containing-protein (SDP) harbors S1 RNA-binding domain. Pentatricopeptide repeat (PPR) protein contains tandem repeats of 35-amino acid motifs. as RZs, have been found in Arabidopsis and rice (Kim et al., 2010a; 2010b; Mangeon et al., 2010). The genomes of Arabidopsis and rice harbor three RZ genes, and AtRZs and OsRZs share approximately $40 \%$ of amino acid sequence similarity as well as the well-conserved CCHC-type zinc finger motif (Fig. 1).

Cold shock domain (CSD) is found in Y-box proteins in eukaryotes and has the ability to bind RNA, single-stranded DNA, and double-stranded DNA (Graumann and Marahiel, 1998; Manival et al., 2001). Contrary to bacterial cold shock protein (CSP) that contains only the CSD, typical plant CSDPs harbor a C-terminal glycine-rich region interspersed with several CCHC-type zinc fingers, in addition to CSD at the N-terminus (Chaikam and Karlson, 2008; Karlson and Imai, 2003; Karlson et al., 2002) (Fig. 1). Four genes encoding CSDPs have been identified in Arabidopsis and rice genomes (Karlson and Imai, 2003). RHs are classified into six superfamilies (SF1-SF6), and each RH has well-defined nine motifs $(\mathrm{Q}, \mathrm{I}$, la, Ib, II, III, IV, V, and $\mathrm{VI}$ ) (Fig. 1). Among the RHs, DEAD-box RHs are the largest family in SF2 helicases that possess the conserved $Q$ motif and helicase activity. The Arabidopsis and rice genomes encode approximately 58 and 50 DEAD-box RHs, respectively (Mingam et al., 2004).

CRM proteins were first investigated in prokaryotes, such as archaea and bacteria, and are divided into four subfamilies, depending on their sequence similarity and domain structures (Barkan et al., 2007). CRM domains are orthologous to the bacterial YhbY and are similar to KH RNA-binding domain that contains a highly conserved GxxG sequence in the loop of CRM domain (Barkan et al., 2007; Keren et al., 2008; Ostheimer et al., 2002) (Fig. 1). Many CRM domain-containing proteins are targeted to chloroplasts or mitochondria and play important roles in the splicing of group I or group II introns in plants (Asakura et al., 2008; Kroeger et al., 2009; Ostersetzer et al., 2005; Zmudjak et al., 2013).

SDPs possess the S1 RNA-binding domain that has RNAbinding ability, which participates in RNA degradation and protein synthesis (Aliprandi et al., 2008; Delvillani et al., 2011). The E. coli S1 protein consists of six S1 domains folded into five-stranded antiparallel $\beta$ barrel, which are involved in RNA binding (Bycroft et al., 1997) (Fig. 1). SDPs are widely present in diverse living organisms, but the functional roles of only a few SDPs have been investigated in plants, including RNA processing and degradation by RNase E/G type endoribonuclease and exosome subunit (AtRrp4p) and plastid transcrip- tion (Chekanova et al., 2002; Jeon et al., 2012; Schein et al. 2008).

PPR proteins consist of tandem repeats of 35-amino acid motifs that fold into one pair of antiparallel $\alpha$ helices (SchmitzLinneweber and Small, 2008; Small and Peeters, 2000; Yin et al., 2013) (Fig. 1). Importantly, PPR proteins are abundant in land plants and mainly localized in organelles such as chloroplasts or mitochondria (de Longevialle et al., 2010; Lurin et al., 2004; O'Toole et al., 2008; Saha et al., 2007). Plant PPR proteins are divided into two major subfamilies of P- and PLSclasses ( $P$ motif: 35 amino acids, L motif: 36 amino acids, and $S$ motif: 31 amino acids) and are involved in RNA metabolism via recognizing specific sequences of RNA substrates (Barkan and Small, 2014; Filipovska and Rackham, 2012; Schmitz-Linneweber and Small, 2008). Proteome analysis revealed that the Arabidopsis and rice genomes encode more than 450 and 650 PPR proteins, respectively, whereas prokaryotes and humans have less than 7 PPR proteins (Lightowlers and Chrzanowska-Lightowlers, 2008; Rackham and Filipovska, 2012).

\section{ROLES OF RBPS IN PLANT GROWTH AND DEVELOPMENT}

Recent years have seen an increase in the assessment of the functional roles of RBPs related to the regulation of flowering time and floral organ formation (Figure 2). FCA and FPA harboring two RRMs enhance flowering by preventing the expression of floral repressor FLOWERING LOCUS C (FLC) (Macknight et al., 1997; Lim et al., 2004). AtGRP7 and KH domain proteins such as PEPPER and FLOWERING LOCUS $\mathrm{K}$ promote flowering by inhibiting FLC expression (Lim et al., 2004; Mockler et al., 2004; Ripoll et al., 2006). It was demonstrated that AtGRP2 is involved in seed and flower development (Fusaro et al., 2007). In addition, RH SWA3 is necessary for embryogenesis or embryo development in Arabidopsis (Liu et al., 2010a; Tripurani et al., 2011). Recently, the importance of RBPs in intron splicing and plant growth and development has been demonstrated. Among the seven minor spliceosomal small nuclear ribonucleoproteins that are involved in splicing of U12-type introns, U11/ U12-31K and U11/U12-65K proteins play essential roles in the splicing of many $U 12$ introns, which is crucial for normal growth and development of dicot and monocot plants (Jung and Kang, 2014; Kim et al., 2010c; Kwak et al., 2012). 


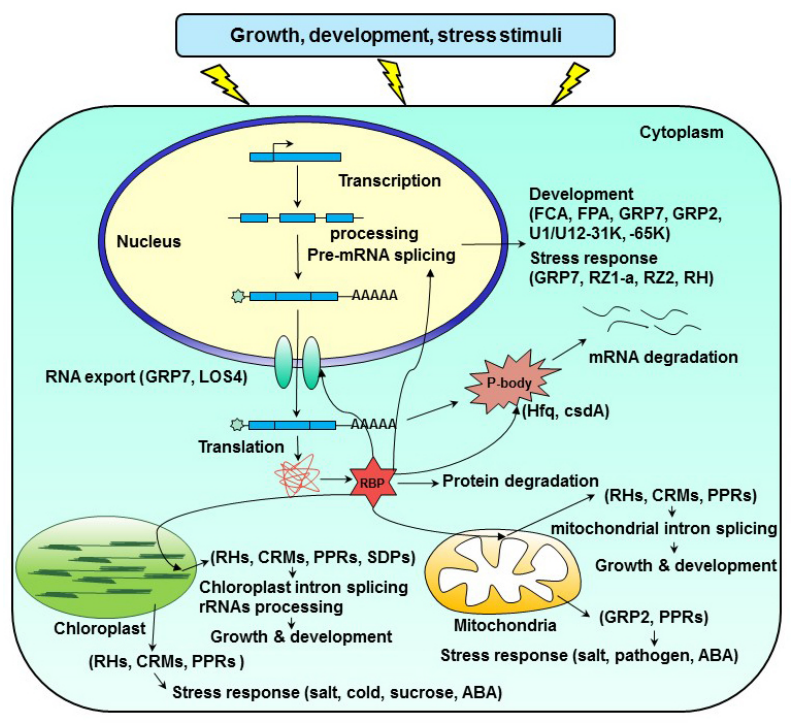

Gene expression in chloroplasts and mitochondria is mainly regulated at posttranscriptional levels such as intron splicing, RNA editing, and RNA degradation, which is crucial for the biogenesis and function of chloroplasts and mitochondria (del Campo, 2009; Stern et al., 2010). Many recent studies have demonstrated that a variety of nuclear-encoded RBPs are targeted to chloroplasts or mitochondria and play indispensable roles during RNA metabolism in these cellular organelles (del Campo, 2009; Stern et al., 2010). The chloroplast-targeted DEAD-box RHs, AtRH3, AtRH22, and AtRH36, and the mitochondria-targeted $\mathrm{RH}, \mathrm{PMH}$, are associated with the splicing of group II introns and rRNA processing, which affects chloroplast or mitochondria biogenesis during the normal growth and development of plants (Asakura et al., 2012; Chi et al., 2012; Gu et al., 2014; Huang et al., 2010; Kanai et al., 2013; Kohler et al., 2010) (Fig. 2). RBPs harboring CRM domains, including Arabidopsis AtCRS1, AtCAF1, AtCAF2, AtCFM2, AtCFM3, and AtCFM4, are involved in the splicing of specific introns as well as the processing of $16 \mathrm{~S}$ and $23 \mathrm{~S}$ rRNAs (Asakura and Barkan, 2006; 2007; Asakura et al., 2008; Lee et al., 2014), while the mitochondria-localized mCSF1 is involved in the splicing of multiple mitochondrial introns (Zmudjak et al., 2013) (Fig. 2). Importantly, most CRM proteins play crucial roles in intron splicing or processing of the genes that are associated with photosystems or respiratory-related complexes, which affects embryogenesis as well as chloroplast and mitochondrial biogenesis. Recently, several SDP proteins, including STF in Nicotiana benthamiana and SDP and SRRP1 in Arabidopsis thaliana, have been determined to play roles in plastid gene expression and rRNA processing during plant growth (Gu et al., 2015; Han et al., 2015; Jeon et al., 2012) (Fig. 2). Although PPR proteins are prevalent in land plants, and plant genomes harbor $>400$ PPR protein-encoding genes, the importance and functional roles of only a small number of PPR proteins have been determined in Arabidopsis, rice, and maize. Maize PPR proteins PPR4 and PPR5 and Arabidopsis PPR proteins OTP51 and OTP70 are crucial for the splicing of chloroplast introns (Chateigner-Boutin et al., 2011; de Longevialle et al., 2008; Schmitz-Linneweber et al., 2006), while Arabidopsis PPR proteins OTP43, BIR6, ABO5, and TANG2 are essential for splicing of mitochondrial introns (des Francs-Small et al., 2014; Koprivova et al., 2010; Liu et al., 2010b; de Longevialle et al.,
Fig. 2. Cellular functions of diverse RNA-binding proteins involved in RNA metabolism during growth, development, and stress response of plants. A variety of RNA-binding proteins (RBPs) play essential roles in RNA processing and pre-mRNA splicing in the nucleus, RNA export, mRNA degradation, and translational control in the cytoplasm. Moreover, diverse nuclear-encoded RBPs are targeted to mitochondria or chloroplasts and play indispensable roles in mitochondria or chloroplasts RNA metabolism, which is crucial for organellar biogenesis and function. Examples of RBPs whose functions in each cellular process have been experimentally determined are shown in parenthesis. Abbreviations such as CRM, GRP, PPR, RZ, $\mathrm{RH}$, and SDP are described in Fig. 1.

2007) (Fig. 2). These results clearly demonstrate that PPRs play indispensable roles during organellar RNA metabolism, which is essential for normal growth and development of plants.

\section{DIVERSE ROLES OF RBPS IN ABIOTIC STRESS RESPONSES}

The involvement of RBPs in the response of plants to different environmental cues has been consistently demonstrated in diverse plant species (Lorković, 2009; Mangeon et al., 2010; Sachetto-Martins, 2000). In particular, the stress-responsive expression and functional roles of RBPs, including GRPs, RZs, CSDPs, RHs, CRMs, SDPs, and PPRs, have been extensively investigated in Arabidopsis, rice, wheat (Triticum aestivum), barely (Hordeum vulgare), and cabbage (Brassica rapa) under diverse environmental conditions (Fig. 2). Arabidopsis AtGRP2 and AtGRP7 and rice OsGRP1 and OsGRP4 promote seed germination and seedling growth under low temperatures and confer freezing tolerance to Arabidopsis plants (Kim et al., 2007a; 2008a; 2010a; Kwak et al., 2005). Interestingly, among the three RZ family members present in the Arabidopsis, rice, and wheat genomes, only AtRZ-1a, OsRZ2, and TaRZ2 affect seed germination and seedling growth at low and freezing temperatures (Kim and Kang, 2006; Kim et al., 2005; 2010b; Xu et al., 2014). These studies demonstrate that certain family members of GRPs and RZs play important roles in plant response to abiotic stresses.

Plant CSDPs are highly induced in plants upon stress treatment, especially by cold stress treatment. The Arabidopsis and rice genomes harbor four genes encoding CSDPs with different number of CCHC-type zinc fingers (Karlson and Imai, 2003; Karlson et al., 2002). Among the four CSDPs found in Arabidopsis, AtCSDP2 and AtCSDP3 confer freezing tolerance to Arabidopsis (Kim et al., 2009; Sasaki et al., 2007) (Fig. 2). Moreover, rice and wheat CSDPs are involved in the cold adaptation process (Chaikam and Karlson, 2008; Nakaminami et al., 2006). Ectopic expression of bacterial CSP in plants enhances cold adaptation in Arabidopsis, rice, and maize (Castiglioni et al., 2008), suggesting functional conservation of CSDPs between plants and bacteria.

DEAD-box RHs can catalyze the unwinding of the secondary structures in RNA molecules and thereby affect RNA metabo- 
lism in living organisms (Jankowsky, 2011). Although the plant genomes harbor more than 50 DEAD-box RHs (Mingam et al., 2004), the functional roles of RHs have been determined for only a limited number of RH family members (Fig. 2). Arabidopsis AtRH38 enhances freezing tolerance (Gong et al., 2002; 2005), and Arabidopsis STRS1 and STRS2 are involved in plant response to multiple abiotic stresses (Kant et al., 2007). Overexpression of Arabidopsis AtRH9 and AtRH25 delays seed germination under high salt conditions, whereas overexpression of AtRH25 enhances freezing tolerance (Kim et al., 2008b). Recently, it has been demonstrated that chloroplast-targeted AtRH3 affects intron splicing of chloroplast $n d h A$ and $n d h B$ genes under salt and cold stresses (Gu et al., 2014). The coldinducible $\mathrm{RH}, \mathrm{RCF} 1$, is indispensable for pre-mRNA splicing and regulation of cold-responsive genes in plants (Guan et al., 2013). Despite the fact that the functional roles of most RHs have not yet been determined, these results certainly suggest that DEAD-box RHs are essential in plant adaptation to diverse abiotic stresses.

Several recent studies have demonstrated that chloroplastor mitochondria-targeted RBPs play an important role in plant response to environmental stimuli as well as plant growth and development under normal conditions (Fig. 2). The chloroplasttargeted CFM4 harboring a single CRM domain was determined to promote seed germination and seedling growth of Arabidopsis under cold and salt stress conditions (Lee et al., 2014). The chloroplast-targeted S1 domain protein SRRP1 is involved in intron splicing of chloroplast tRNA and affects seedling growth of Arabidopsis in the presence of abscisic acid (ABA) (Gu et al., 2015). Contrary to the ample studies elucidating the functional roles of PPRs in plant growth and development under normal conditions, reports demonstrating the roles of PPRs in plant stress responses are severely limited. The chloroplast-targeted Arabidopsis PPR protein GUN1 enhances seedling development in the presence of sucrose and ABA, and the chloroplast-targeted rice PPR protein OsV4 is required for chloroplast development during the cold adaptation process (Cottage et al., 2010; Gong et al., 2014). Arabidopsis SOAR1 and rice WSL have been determined to play important roles in plant response to multiple environmental stimuli, including $A B A$, drought, salt, sucrose, and cold (Jiang et al., 2015; Mei et al., 2014; Tan et al., 2014). Overexpression of mitochondriatargeted PPR40 in Arabidopsis was shown to promote seed germination and seedling growth of the plants under salt stress, whereas the ppr40 mutant showed enhanced sensitivity to abiotic stresses such as high salinity, oxidative stress, and ABA (Zsigmond et al., 2008; 2012). Moreover, it has been demonstrated that Arabidopsis mitochondria-targeted PGN plays an essential role in plant defense against fungi as well as in plant tolerance against abiotic stress (Laluk et al., 2011). All of these reports emphasize that diverse chloroplast- or mitochondriatargeted RBPs play an essential role in organellar RNA metabolism, biogenesis, and function under stressful conditions as well as under normal growth conditions.

\section{CELLULAR ROLES OF RBPS AS RNA CHAPERONES}

Contrary to the increasing understanding of the importance and emerging roles of RBPs in plant growth, development, and stress responses, the mechanistic roles of RBPs in these cellular processes are largely unknown. However, several recent studies point to the cellular roles of RBPs as RNA chaperones in plant growth, development, and stress responses. During RNA metabolism, RNA molecules need to be correctly folded for normal functioning; however, RNA molecules are easily misfolded into non-functional structures due to their intrinsic kinetic and thermodynamic folding problems (Herschlag, 1995; Woodson, 2010). Both RNA chaperones and specific RBPs can help RNAs to achieve their active functional states (Rajkowitsch et al., 2007). RNA chaperones are nonspecific RBPs that facilitate RNA folding via structural rearrangement of misfolded RNAs (Rajkowitsch et al., 2007; Woodson, 2010). Compared to specific RBPs that bind to specific target RNAs, RNA chaperones generally bind to various RNA substrates with low sequence specificity (Ivanyi-Nagy et al., 2005).

Recent studies have demonstrated that RNA chaperones play crucial roles in the growth and development of living organisms, including bacteria, viruses, animals, and plants (Kang et al., 2013). Some examples of RNA chaperones include viral nucleocapsid protein, long interspersed element-1 in animals, and several bacterial proteins such as $\mathrm{Hfq}$, FinO, and Prop (Arthur et al., 2003; Chambers and Bender, 2011; Chaulk et al., 2011; Martin, 2010). In addition, many E. coli DEAD-box RHs, including DeaD, RhIB, RhIE, and SrmBp, and yeast Mss116p function as an RNA chaperone in the splicing of mitochondrial group I and II introns (Huang et al., 2005; Mohr et al., 2002; 2006). Interestingly, U11/U12-31K, the minor spliceosomal protein, harbors RNA chaperone activity and plays an essential role in the splicing of U12-type introns (Kim et al., 2010c; Kwak et al., 2012). Several recent findings have demonstrated that chloroplast-targeted proteins such as CFM4, RH3, SDP, and SRRP1 possess RNA chaperone activity and affect rRNA processing and intron splicing in chloroplasts ( $\mathrm{Gu}$ et al., 2014, 2015; Han et al., 2015; Lee et al., 2014). These studies clearly indicate that many RBPs harboring RNA chaperone activity play essential roles in the regulation of RNA metabolism during plant growth and development.

Involvement of RBPs with RNA chaperone activity in plant stress responses has been extensively investigated in diverse plant species. It has been determined that Arabidopsis AtCSDP1 and AtCSDP3, cabbage BrCSDP3, wheat CSDPs, and rice CSDPs harbor RNA chaperone ability, which is important for stress response and cold adaptation process in plants (Chaikam and Karlson, 2008; Choi et al., 2015; Kim et al., 2007a, 2009). In addition, many GRP and RZ family members, including Arabidopsis AtGRP2 and AtGRP7, rice OsGRP1, OsGRP4 and OsGRP6, Arabidopsis AtRZ-1a, rice OsRZ2, and wheat TaRZ2, possess RNA chaperone activity during stress responses (Kim et al., 2005; 2007b; 2008a; 2010a; 2010b; Xu et al., 2013). Although the functional roles of many RHs in plant stress responses have been demonstrated, the RNA chaperone activity of only AtRH25 was determined in Arabidopsis under freeze stress conditions (Kim et al., 2008b). Considering that PPR proteins are widely present in land plants and are involved in chloroplast or mitochondrial RNA metabolism under normal and stress conditions, it would be interesting to determine whether any PPR proteins display RNA chaperone function in plants under diverse environmental stimuli.

\section{CONCLUSION}

Although studies on the functional roles of RBPs in growth, development, and stress response of plants are rapidly increasing in recent years, our understanding of the cellular roles of RBPs during RNA metabolism in plants is far from sufficient. Considering that a variety of RBPs are targeted to chloroplasts and/or mitochondria, it would be of interest to determine the functional roles of these RBPs in organellar RNA metabolism during plant growth and development as well as plant stress responses. In particular, investigation of the RNA chaperone 
activity of RBPs will provide clues about the cellular roles and action mechanism of RBPs during RNA metabolism in organelle biogenesis, plant development, and stress responses. As recent development of RNA-seq allows us to investigate the genome-wide analysis of RNAs in an organism, it would be of great interest to determine the fates and processing of RNAs in the mutants of particular RBPs involved in plant growth and stress responses. A major task for the future is to identify RNA targets and to understand how RBPs recognize substrate RNAs and how RBPs interact with other protein factors to regulate posttranscriptional RNA metabolism during plant growth and development under normal as well as stressful conditions.

\section{ACKNOWLEDGMENTS}

This work was supported by the Mid-career Researcher Program through the National Research Foundation of Korea, the Ministry of Education, Science and Technology (2011-0017357), and the Next-Generation BioGreen21 Program (PJ01105401), Rural Development Administration, Republic of Korea.

\section{REFERENCES}

Alba, M.M., and Pages, M. (1998). Plant proteins containing the RNA-recognition motif. Trends Plant Sci. 3, 15-21.

Aliprandi, P., Sizun, C., Perez, J., Mareuil, F., Caputo, S., Leroy, J.L., Odaert, B., Laalami, S., Uzan, M., and Bontems, F. (2008). S1 ribosomal protein functions in translation initiation and ribonuclease RegB activation are mediated by similar RNAprotein interactions: an NMR and SAXS analysis. J. Biol. Chem. 283, 13289-13301.

Arthur, D.C., Ghetu, A.F., Gubbins, M.J., Edwards, R.A., Frost, L.S., and Glover, J.M. (2003). FinO is an RNA chaperone that facilitates sense-antisense RNA interactions. EMBO J. 22, 6346-6355.

Asakura, Y., and Barkan, A. (2006). Arabidopsis orthologs of maize chloroplast splicing factors promote splicing of orthologous and species-specific group II introns. Plant Physiol. 142, 1656-1663.

Asakura, Y., and Barkan, A. (2007). A CRM domain protein functions dually in group I and group II intron splicing in land plant chloroplasts. Plant Cell 19, 3864-3875.

Asakura, Y., Bayraktar, O.A., and Barkan, A. (2008). Two CRM protein subfamilies cooperate in the splicing of group IIB introns in chloroplasts. RNA 14, 2319-2332.

Asakura, Y., Galarneau, E., Watkins, K.P., Barkan, A., and van Wijk, K.J. (2012). Chloroplast RH3 DEAD Box RNA helicases in maize and Arabidopsis function in splicing of specific group II introns and affect chloroplast ribosome riogenesis. Plant Physiol. 159, 961-974.

Barkan, A., and Small, I. (2014). Pentatricopeptide repeat proteins in plants. Annu. Rev. Plant Biol. 65, 415-442.

Barkan, A., Klipcan, L., Ostersetzer, O., Kawamura, T., Asakura, Y., and Watkins, K.P. (2007). The CRM domain: an RNA binding module derived from an ancient ribosome-associated protein. RNA 13, 55-64.

Brown, G.G., Colas des Francs-Small, C., and Ostersetzer-Biran, O. (2014). Group II intron splicing factors in plant mitochondria. Front. Plant Sci. 5, 35

Bycroft, M., Hubbard, T.J., Proctor, M., Freund, S.M., and Murzin, A.G. (1997). The solution structure of the S1 RNA binding domain: A member of an ancient nucleic acid-binding fold. Cell $88,235-242$

del Campo, E.M. (2009). Post-transcriptional control of chloroplast gene expression. Gene Regul. Syst. Biol. 3, 31.

Castiglioni, P., Warner, D., Bensen, R.J., Anstrom, D.C., Harrison, J., Stoecker, M., Abad, M., Kumar, G., Salvador, S., D'Ordine, R., et al. (2008). Bacterial RNA chaperones confer abiotic stress tolerance in plants and improved grain yield in maize under water-limited conditions. Plant Physiol. 147, 446-455.

Chaikam, V., and Karlson, D. (2008). Functional characterization of two cold shock domain proteins from Oryza sativa. Plant Cell Environ. 31, 995-1006.

Chaulk, S., Smith Frieday, M.N., Arthur, D.C., Culham, D.E.,
Edwards, R.A., Soo, P., Frost, L.S., Keates, R.A., Glover, J.M. and Wood, J.M. (2011). ProQ is an RNA chaperone that controls ProP levels in Escherichia coli. Biochemistry 50, 30953106.

Chambers, J.R., and Bender, K.S. (2011). The RNA chaperone Hfa is important for growth and stress tolerance in Francisella novicida. PLoS One 6, e19797.

Chateigner-Boutin, A.L., des Francs-Small, C.C., Delannoy, E., Kahlau, S., Tanz, S.K., de Longevialle, A.F., Fujii, S., and Small, I. (2011). OTP70 is a pentatricopeptide repeat protein of the $E$ subgroup involved in splicing of the plastid transcript rpoC1. Plant J. 65, 532-542.

Chekanova, J.A., Dutko, J.A., Mian, I.S., and Belostotsky, D.A. (2002). Arabidopsis thaliana exosome subunit AtRrp4p is a hydrolytic $3^{\prime} \rightarrow 5^{\prime}$ exonuclease containing $\mathrm{S} 1$ and $\mathrm{KH}$ RNAbinding domains. Nucleic Acids Res. 30, 695-700.

Chi, W., He, B., Mao, J., Li, Q., Ma, J., Ji, D., Zou, M., and Zhang, L. (2012). The function of RH22, a DEAD RNA helicase, in the biogenesis of the $50 \mathrm{~S}$ ribosomal subunits of Arabidopsis chloroplasts. Plant Physiol. 158, 693-707.

Choi, M.J., Park, Y.R., Park, S.J., and Kang, H. (2015). Stressresponsive expression patterns and functional characterization of cold shock domain proteins in cabbage (Brassica rapa) under abiotic stress conditions. Plant Physiol. Biochem. 96, 132-140.

Cottage, A., Mott, E.K., Kempster, J.A., and Gray, J.C. (2010). The Arabidopsis plastid-signalling mutant gun1 (genomes uncoupled1) shows altered sensitivity to sucrose and abscisic acid and alterations in early seedling development. J. Exp. Bot. 61, 3773-3786

Delvillani, F., Papiani, G., Dehò, G., and Briani, F. (2011). S1 ribosomal protein and the interplay between translation and mRNA decay. Nucleic Acids Res. 39, 7702-7715.

des Francs-Small, C.C., de Longevialle, A.F., Li, Y., Lowe, E., Tanz, S.K., Smith, C., Bevan, M.W., and Small, I. (2014). The pentatricopeptide repeat proteins TANG2 and ORGANELLE TRANSCRIPT PROCESSING439 are involved in the splicing of the multipartite nad5 transcript encoding a subunit of mitochondrial Complex I. Plant Physiol. 165, 1409-1416.

Filipovska, A., and Rackham, O. (2012). Modular recognition of nucleic acids by PUF, TALE and PPR proteins. Mol. Biosyst. 8 , 699-708.

Floris, M., Mahgoub, H., Lanet, E., Robaglia, C., and Menand, B. (2009). Post-transcriptional Regulation of Gene Expression in Plants during Abiotic Stress. Int. J. Mol. Sci. 10, 3168-3185.

Fusaro, A.F., Bocca, S.N., Ramos, R.L.B., Barrôco, R.M., Magioli, C., Jorge, V.C., Coutinho, T.C., Rangel-Lima, C.M., De Rycke, R., and Inzé, D. (2007). AtGRP2, a cold-induced nucleocytoplasmic RNA-binding protein, has a role in flower and seed development. Planta 225, 1339-1351

Gong, Z., Lee, H., Xiong, L., Jagendorf, A., Stevenson, B., and Zhu, J.-K. (2002). RNA helicase-like protein as an early regulator of transcription factors for plant chilling and freezing tolerance. Proc. Natl. Acad. Sci. USA 99, 11507-11512.

Gong, Z., Dong, C.-H., Lee, H., Zhu, J., Xiong, L., Gong, D. Stevenson, B., and Zhu, J.-K. (2005). A DEAD box RNA helicase is essential for mRNA export and important for development and stress responses in Arabidopsis. Plant Cell 17, 256-267.

Gong, X.D., Su, Q.Q., Lin, D.Z., Jiang, Q., Xu, J.L., Zhang, J.H., Teng, S., and Dong, Y.J. (2014). The rice OsV4 encoding a novel pentatricopeptide repeat protein is required for chloroplast development during the early leaf stage under cold stress. J. Integr. Plant Biol. 56, 400-410.

Graumann, P.L., and Marahiel, M.A. (1998). A superfamily of proteins that contain the cold-shock domain. Trends Biochem. Sci. 23, 286-290.

Gu, L., Xu, T., Lee, K., Lee, K.H., and Kang, H. (2014). A chloroplast-localized DEAD-box RNA helicaseAtRH3 is essential for intron splicing and plays an important role in the growth and stress response in Arabidopsis thaliana. Plant Physiol. Biochem. 82, 309-318.

Gu, L., Jung, H.J., Kim, B.M., Xu, T., Lee, K., Kim, Y.O., and Kang, H. (2015). A chloroplast-localized S1 domain-containing protein SRRP1 plays a role in Arabidopsis seedling growth in the presence of ABA. J. Plant. Physiol. 189, 34-41.

Guan, Q., Wu, J., Zhang, Y., Jiang, C., Liu, R., Chai, C., and Zhu, J. 
(2013). A DEAD box RNA helicase is critical for pre-mRNA splicing, cold-responsive gene regulation, and cold tolerance in Arabidopsis. Plant Cell 25, 342-356.

Hammani, K., and Giege, P. (2014). RNA metabolism in plant mitochondria. Trends Plant Sci. 19, 380-389.

Han, J.H., Lee, K., Lee, K.H., Jung, S., Jeon, Y., Pai, H.S., and Kang, H. (2015). A nuclear-encoded chloroplast-targeted S1 RNA-binding domain protein affects chloroplast rRNA processing and is crucial for the normal growth of Arabidopsis thaliana. Plant J. 83, 277-289.

Herschlag, D. (1995). RNA chaperones and the RNA folding problem. J. Biol. Chem. 270, 20871-20874.

Huang, H.-R., Rowe, C.E., Mohr, S., Jiang, Y., Lambowitz, A.M., and Perlman, P.S. (2005). The splicing of yeast mitochondrial group I and group II introns requires a DEAD-box protein with RNA chaperone function. Proc. Natl. Acad. Sci. USA 102, 163168.

Huang, C.-K., Huang, L.-F., Huang, J.-J., Wu, S.-J., Yeh, C.-H., and Lu, C.-A. (2010). A DEAD-box protein, AtRH36, is essential for female gametophyte development and is involved in rRNA biogenesis in Arabidopsis. Plant Cell Physiol. 51, 694-706.

Ivanyi-Nagy, R., Davidovic, L., Khandjian, E., and Darlix, J.-L. (2005). Disordered RNA chaperone proteins: from functions to disease. Cell. Mol. Life Sci. 62, 1409-1417.

Jankowsky, E. (2011). RNA helicases at work: binding and rearranging. Trends Biochem. Sci. 36, 19-29.

Jeon, Y., Jung, H.J., Kang, H., Park, Y.I., Lee, S.H., and Pai, H.S. (2012). S1 domain-containing STF modulates plastid transcription and chloroplast biogenesis in Nicotiana benthamiana. New Phytol. 193, 349-363.

Jiang, S.-C., Mei, C., Liang, S., Yu, Y.-T., Lu, K., Wu, Z., Wang, X.F., and Zhang, D.-P. (2015). Crucial roles of the pentatricopeptide repeat protein SOAR1 in Arabidopsis response to drought, salt and cold stresses. Plant Mol. Biol. 88, 369-385.

Jung, H.J., and Kang, H. (2014). The Arabidopsis U11/U12-65K is an indispensable component of minor spliceosome and plays a crucial role in U12 intron splicing and plant development. Plant J. 78, 799-810.

Jung, H.J., Park, S.J., and Kang, H.S., (2013). Regulation of RNA metabolism in plant development and stress responses. J. Plant Biol. 56, 123-129.

Kanai, M., Hayashi, M., Kondo, M., and Nishimura, M. (2013). The plastidic DEAD-box RNA helicase 22, HS3, is essential for plastid functions both in seed development and in seedling growth. Plant Cell Physiol. 54, 1431-1440.

Kang, H., Park, S.J., and Kwak, K.J. (2013). Plant RNA chaperones in stress response. Trends Plant Sci. 18, 100-106.

Kant, P., Kant, S., Gordon, M., Shaked, R., and Barak, S. (2007). STRESS RESPONSE SUPPRESSOR1 and STRESS RESPONSE SUPPRESSOR2, two DEAD-box RNA helicases that attenuate Arabidopsis responses to multiple abiotic stresses. Plant Physiol. 145, 814-830.

Karlson, D., and Imai, R. (2003). Conservation of the cold shock domain protein family in plants. Plant Physiol. 131, 12-15

Karlson, D., Nakaminami, K., Toyomasu, T., and Imai, R. (2002). A cold-regulated nucleic acid-binding protein of winter wheat shares a domain with bacterial cold shock proteins. J. Biol. Chem. 277, 35248-35256.

Keren, I., Klipcan, L., Bezawork-Geleta, A., Kolton, M., Shaya, F. and Ostersetzer-Biran, O. (2008). Characterization of the molecular basis of group II intron RNA recognition by CRS1CRM domains. J. Biol. Chem. 283, 23333-23342.

Kim, Y.O., and Kang, H. (2006). The role of a zinc finger-containing glycine-rich RNA-binding protein during the cold adaptation process in Arabidopsis thaliana. Plant Cell Physiol. 47, 793-798.

Kim, Y.O., Kim, J.S., and Kang, H. (2005). Cold-inducible zinc finger-containing glycine-rich RNA-binding protein contributes to the enhancement of freezing tolerance in Arabidopsis thaliana. Plant J. 42, 890-900.

Kim, J.S., Park, S.J., Kwak, K.J., Kim, Y.O., Kim, J.Y., Song, J., Jang, B., Jung, C.H., and Kang, H. (2007a). Cold shock domain proteins and glycine-rich RNA-binding proteins from Arabidopsis thaliana can promote the cold adaptation process in Escherichia coli. Nucleic Acids Res. 35, 506-516.

Kim, J.Y., Park, S.J., Jang, B., Jung, C.H., Ahn, S.J., Goh, C.H.,
Cho, K., Han, O., and Kang, H. (2007b). Functional characterization of a glycine-rich RNA-binding protein 2 in Arabidopsis thaliana under abiotic stress conditions. Plant J. 50, 439-451.

Kim, J.S., Jung, H.J., Lee, H.J., Kim, K., Goh, C.H., Woo, Y., Oh, S.H., Han, Y.S., and Kang, H. (2008a). Glycine-rich RNA-binding protein7 affects abiotic stress responses by regulating stomata opening and closing in Arabidopsis thaliana. Plant J. 55, 455-466.

Kim, J.S., Kim, K.A., Oh, T.R., Park, C.M., and Kang, H. (2008b). Functional Characterization of DEAD-Box RNA Helicases in Arabidopsis thaliana under Abiotic Stress Conditions. Plant Cell Physiol. 49, 1563-1571.

Kim, M-H. Sasaki, K and Imai, R. (2009). Cold shock domain protein 3 regulates freezing tolerance in Arabidopsis thaliana. $\mathrm{J}$. Biol. Chem. 284, 23454-23460.

Kim, J.Y., Kim, W.Y., Kwak, K.J., Oh, S.H., Han, Y.S., and Kang, H. (2010a). Glycine-rich RNA-binding proteins are functionally conserved in Arabidopsis thaliana and Oryza sativa during cold adaptation process. J. Exp. Bot. 61, 2317-2325.

Kim, J.Y., Kim, W.Y., Kwak, K.J., Oh, S.H., Han, Y.S., and Kang, H. (2010b). Zinc finger-containing glycine-rich RNA-binding protein in Oryza sativa has an RNA chaperone activity under cold stress conditions. Plant Cell Environ. 33, 759-768.

Kim, W.Y., Jung, H.J., Kwak, K.J., Kim, M.K., Oh, S.H., Han, Y.S., and Kang, H. (2010c). The Arabidopsis U12-type spliceosomal protein U11/U12-31K is involved in U12 intron splicing via RNA chaperone activity and affects plant development. Plant Cell 22, 3951-3962.

Kohler, D., Schmidt-Gattung, S., and Binder, S. (2010). The DEADbox protein $\mathrm{PMH} 2$ is required for efficient group II intron splicing in mitochondria of Arabidopsis thaliana. Plant. Mol. Biol. 72, 459467

Koprivova, A., des Francs-Small, C.C., Calder, G., Mugford, S.T., Tanz, S., Lee, B.R., Zechmann, B., Small, I., and Kopriva, S (2010). Identification of a pentatricopeptide repeat protein implicated in splicing of intron 1 of mitochondrial nad7 transcripts. J. Biol Chem 285, 32192-32199.

Kroeger, T.S., Watkins, K.P., Friso, G., van Wijk, K.J., and Barkan, A. (2009). A plant-specific RNA-binding domain revealed through analysis of chloroplast group II intron splicing. Proc. Natl. Acad. Sci. USA 106, 4537-4542.

Kwak, K.J., Kim, Y.O., and Kang, H. (2005). Characterization of transgenic Arabidopsis plants overexpressing GR-RBP4 under high salinity, dehydration, or cold stress. J. Exp. Bot. 56, 30073016.

Kwak, K.J., Jung, H.J., Lee, K.H., Kim, Y.S., Kim, W.Y., Ahn, S.J., and Kang, H. (2012). The minor spliceosomal protein U11/U12$31 \mathrm{~K}$ is an RNA chaperone crucial for U12 intron splicing and the development of dicot and monocot plants. PLoS One 7, e43707.

Laluk, K., AbuQamar, S., and Mengiste, T. (2011). The Arabidopsis mitochondria-localized pentatricopeptide repeat protein PGN functions in defense against necrotrophic fungi and abiotic stress tolerance. Plant Physiol. 156, 2053-2068

Lee, K., Lee, H.J., Kim, D.H., Jeon, Y., Pai, H.S., and Kang, H. (2014). A nuclear-encoded chloroplast protein harboring a single CRM domain plays an important role in the Arabidopsis growth and stress response. BMC Plant Biol. 14, 98

Lightowlers, R., and Chrzanowska-Lightowlers, Z. (2008). PPR (pentatricopeptide repeat) proteins in mammals: important aids to mitochondrial gene expression. Biochem. J. 416, e5-e6.

Lim, M.-H., Kim, J., Kim, Y.-S., Chung, K.-S., Seo, Y.-H., Lee, I., Kim, J., Hong, C.B., Kim, H.-J., and Park, C.-M. (2004). A new Arabidopsis gene, FLK, encodes an RNA binding protein with $\mathrm{K}$ homology motifs and regulates flowering time via FLOWERING LOCUS C. Plant Cell 16, 731-740.

Liu, M., Shi, D.Q., Yuan, L., Liu, J., and Yang, W.C. (2010a). SLOW WALKER3, encoding a putative DEAD-box RNA helicase, is essential for female gametogenesis in Arabidopsis. J. Int. Plant Biol. 52, 817-828.

Liu, Y., He, J., Chen, Z., Ren, X., Hong, X., and Gong, Z. (2010b). ABA overly-sensitive 5 (ABO5), encoding a pentatricopeptide repeat protein required for cis-splicing of mitochondrial nad2 intron 3 , is involved in the abscisic acid response in Arabidopsis. Plant J. 63, 749-765.

de Longevialle, A.F., Meyer, E.H., Andres, C., Taylor, N.L., Lurin, C. 
Millar, A.H., and Small, I.D. (2007). The pentatricopeptide repeat gene OTP43 is required for trans-splicing of the mitochondrial nad1 intron 1 in Arabidopsis thaliana. Plant Cell 19, 3256-3265.

de Longevialle, A.F., Hendrickson, L., Taylor, N.L., Delannoy, E., Lurin, C., Badger, M., Millar, A.H., and Small, I. (2008). The pentatricopeptide repeat gene OTP51 with two LAGLIDADG motifs is required for the cis-splicing of plastid ycf3 intron 2 in Arabidopsis thaliana. Plant J. 56, 157-168.

de Longevialle, A.F., Small, I.D., and Lurin, C. (2010). Nuclearly encoded splicing factors implicated in RNA splicing in higher plant organelles. Mol. Plant 3, 691-705.

Lorković, Z.J. (2009). Role of plant RNA-binding proteins in development, stress response and genome organization. Trends Plant Sci.14, 229-236.

Lorković, Z.J., and Barta, A. (2002). Genome analysis: RNA recognition motif (RRM) and $\mathrm{K}$ homology $(\mathrm{KH})$ domain RNAbinding proteins from the flowering plant Arabidopsis thaliana. Nucleic Acids Res. 30, 623-635.

Macknight, R., Bancroft, I., Page, T., Lister, C., Schmidt, R., Love, K., Westphal, L., Murphy, G., Sherson, S., and Cobbett, C. (1997). FCA, a gene controlling flowering time in Arabidopsis, encodes a protein containing RNA-binding domains. Cell 89, 737-745.

Mangeon, A., Junqueira, R.M., and Sachetto-Martins, G. (2010). Functional diversity of the plant glycine-rich proteins superfamily. Plant Signal. Behav. 5, 99-104.

Manival, X., Ghisolfi-Nieto, L., Joseph, G., Bouvet, P., and Erard, M. (2001). RNA-binding strategies common to cold-shock domainand RNA recognition motif-containing proteins. Nucleic Acids Res. 29, 2223-2233.

Martin, S.L. (2010). Nucleic acid chaperone properties of ORF1p from the non-LTR retrotransposon, LINE-1. RNA Biol. 7, 706711.

Mei, C., Jiang, S.-C., Lu, Y.-F., Wu, F.-Q., Yu, Y.-T., Liang, S., Feng, X.-J., Comeras, S.P., Lu, K., and Wu, Z. (2014). Arabidopsis pentatricopeptide repeat protein SOAR1 plays a critical role in abscisic acid signalling. J. Exp. Bot. 65, 5317-5330.

Mihailovich, M., Militti, C., Gabaldon, T., and Gebauer, F. (2010). Eukaryotic cold shock domain proteins: highly versatile regulators of gene expression. BioEssays 32, 109-118.

Mingam, A., Toffano-Nioche, C., Brunaud, V., Boudet, N., Kreis, M., and Lecharny, A. (2004). DEAD-box RNA helicases in Arabidopsis thaliana: establishing a link between quantitative expression, gene structure and evolution of a family of genes. J. Plant Biotechnol. 2, 401-415.

Mockler, T.C., Yu, X., Shalitin, D., Parikh, D., Michael, T.P., Liou, J., Huang, J., Smith, Z., Alonso, J.M., and Ecker, J.R. (2004). Regulation of flowering time in Arabidopsis by $\mathrm{K}$ homology domain proteins. Proc. Natl. Acad. Sci. USA 101, 12759-12764.

Mohr, S., Stryker, J.M., and Lambowitz, A.M. (2002). A DEAD-box protein functions as an ATP-dependent RNA chaperone in group I intron splicing. Cell 109, 769-779.

Mohr, S., Matsuura, M., Perlman, P.S., and Lambowitz, A.M. (2006) A DEAD-box protein alone promotes group II intron splicing and reverse splicing by acting as an RNA chaperone. Proc. Natl. Acad. Sci. U.S.A. 103, 3569-3574.

Nagai, K., Oubridge, C., Ito, N., Avis, J., and Evans, P. (1995). The RNP domain : a sequence- specific RNA-binding domain involved in processing and transport of RNA. Trends Biochem. Sci. 20, 235-240.

Nakaminami, K., Karlson, D.T., and Imai, R. (2006). Functional conservation of cold shock domains in bacteria and higher plants. Proc. Natl. Acad. Sci. U.S.A. 103, 10122-10127.

O'Toole, N., Hattori, M., Andres, C., lida, K., Lurin, C., SchmitzLinneweber, C., Sugita, M., and Small, I. (2008). On the expansion of the pentatricopeptide repeat gene family in plants. Mol. Biol. Evol. 25, 1120-1128.

Ostersetzer, O., Cooke, A.M., Watkins, K.P., and Barkan, A. (2005). CRS1, a chloroplast group II intron splicing factor, promotes intron folding through specific interactions with two intron domains. Plant Cell 17, 241-255.

Ostheimer, G.J., Barkan, A., and Matthews, B.W. (2002). Crystal structure of E. coli YhbY: a representative of a novel class of RNA binding proteins. Structure 10,1593-1601.

Rackham, O., and Filipovska, A. (2012). The role of mammalian PPR domain proteins in the regulation of mitochondrial gene expression. Biochim. Biophys. Acta 1819, 1008-1016.

Rajkowitsch, L., Chen, D., Stampfl, S., Semrad, K., Waldsich, C., Mayer, O., Jantsch, M.F., Konrat, R., Blasi, U., and Schroeder, R. (2007). RNA chaperones, RNA annealers and RNA helicases. RNA Biol. 4, 118-130.

Ripoll, J.J., Ferrándiz, C., Martínez-Laborda, A., and Vera, A (2006). PEPPER, a novel K-homology domain gene, regulates vegetative and gynoecium development in Arabidopsis. Dev. Biol. 289, 346-359.

Sachetto-Martins, G., Franco, L.O., and de Oliveira, D.E. (2000). Plant glycine-rich proteins: a family or just proteins with a common motif? Biochim. Biophys. Acta. 1492, 1-14

Saha, D., Prasad, A.M., and Srinivasan, R. (2007). Pentatricopeptide repeat proteins and their emerging roles in plants. Plant Physiol. Biochem. 45, 521-534

Sasaki, K., Kim, M.-H., and Imai, R. (2007). Arabidopsis COLD SHOCK DOMAIN PROTEIN2 is a RNA chaperone that is regulated by cold and developmental signals. Biochem. Biophys. Res. Commun. 364, 633-638.

Schein, A., Sheffy-Levin, S., Glaser, F., and Schuster, G. (2008). The RNase E/G-type endoribonuclease of higher plants is located in the chloroplast and cleaves RNA similarly to the E. coli enzyme. RNA 14, 1057-1068

Schmitz-Linneweber, C., and Small, I. (2008). Pentatricopeptide repeat proteins: a socket set for organelle gene expression. Trends Plant Sci. 13, 663-670.

Schmitz-Linneweber, C., Williams-Carrier, R.E., Williams-Voelker, P.M., Kroeger, T.S., Vichas, A., and Barkan, A. (2006). A pentatricopeptide repeat protein facilitates the trans-splicing of the maize chloroplast rps12 pre-mRNA. Plant Cell 18, 26502663.

Simpson, G.G., and Filipowicz, W. (1996). Splicing of precursors to mRNA in higher plants: mechanism, regulation and sub-nuclear organisation of the spliceosomal machinery. Plant Mol. Biol. 32, $1-41$

Small, I.D., and Peeters, N. (2000). The PPR motif-a TPR-related motif prevalent in plant organellar proteins. Trends Biochem. Sci. $25,45-47$.

Stern, D.B., Goldschmidt-Clermont, M. and Hanson, M.R. (2010). Chloroplast RNA metabolism. Annu. Rev. Plant Biol. 61, 125155.

Tan, J., Tan, Z., Wu, F., Sheng, P., Heng, Y., Wang, X., Ren, Y., Wang, J., Guo, X., and Zhang, X. (2014). A novel chloroplastlocalized pentatricopeptide repeat protein involved in splicing affects chloroplast development and abiotic stress response in rice. Mol. Plant 7, 1329-1349.

Tripurani, S.K., Nakaminami, K., Thompson, K.B., Crowell, S.V. Guy, C.L., and Karlson, D.T. (2011). Spatial and temporal expression of cold-responsive DEAD-box RNA helicases reveals their functional roles during embryogenesis in Arabidopsis thaliana. Plant Mol. Biol. Rep. 29, 761-768.

Woodson, S.A. (2010). Taming free energy landscapes with RNA chaperones. RNA Biol. 7, 677-686.

Xu, T., Gu, L., Choi, M.J., Kim, R.J., Suh, M.C., and Kang, H. (2014). Comparative functional analysis of wheat (Triticum aestivum). zinc finger-containing glycine-rich RNA-binding proteins in response to abiotic stresses. PLoS One 9, e96877.

Yin, P., Li, Q., Yan, C., Liu, Y., Liu, J., Yu, F., Wang, Z., Long, J., He J., and Wang, H.-W. (2013). Structural basis for the modular recognition of single-stranded RNA by PPR proteins. Nature 504 168-171.

Zmudjak, M., Colas des Francs-Small, C., Keren, I., Shaya, F., Belausov, E., Small, I., and Ostersetzer-Biran, O. (2013). mCSF1, a nucleus-encoded CRM protein required for the processing of many mitochondrial introns, is involved in the biogenesis of respiratory complexes I and IV in Arabidopsis. New Phytol. 199, 379-394.

Zsigmond, L., Rigo, G., Szarka, A., Szekely, G., Otvos, K., Darula, Z., Medzihradszky, K.F., Koncz, C., Koncz, Z., and Szabados, L. (2008). Arabidopsis PPR40 connects abiotic stress responses to mitochondrial electron transport. Plant Physiol. 146, 1721-1737.

Zsigmond, L., Szepesi, A., Tari, I., Rigo, G., Kiraly, A., and Szabados, L. (2012). Overexpression of the mitochondrial PPR40 gene improves salt tolerance in Arabidopsis. Plant Sci. 182, 87-93. 